Equilibrium Contrast-enhanced CT Imaging to Evaluate Hepatic Fibrosis: Initial Validation by Comparison with Histopathologic Analysis ${ }^{1}$

Steve Bandula, FRCR

Shonit Punwani, PhD, FRCR

William M. Rosenberg, DPhil

Rajiv Jalan, MRCP

Andrew R. Hall, MSc, FIBMS

Amar Dhillon, MRCP

James C. Moon, MD

Stuart A. Taylor, MD
Purpose:

Materials and Methods:

Results:

${ }^{1}$ From the UCL Centre for Medical Imaging (S.B., S.P., S.A.T.), UCL Institute for Liver and Digestive Health, Division of Medicine (W.M.R., R.J.), and UCL Institute for Cardiovascular Science (J.C.M.), University College London, Gower St, London WC1E 6BT, England; and Department of Cellular Pathology, UCL Medical School, Royal Free Campus, London, England (A.R.H., A.D.). Received June 18, 2014; revision requested August 1; revision received September 3; accepted October 10; final version accepted October 10 . This article presents independent research funded by the National Institute for Health Research (NIHR). The views expressed are those of the authors and not necessarily those of the NHS, the NIHR, or the Department of Health. W.M.R. and S.A.T. are NIHR Senior Investigators. S.B. is funded by a fellowship from the National Institute for Health Research (NIHRDRF 201104008). The majority of this work was undertaken at University College London Hospital and University College London, which receive a proportion of funding from the NIHR Biomedical Research Centre funding scheme. Address correspondence to S.A.T. (e-mail: stuart.taylor@uclh.nhs.uk).
To prospectively evaluate hepatic extracellular volume (ECV) fraction measurement at equilibrium computed tomographic (CT) imaging compared with both fibrosis quantified with histologic analysis and the enhanced liver fibrosis panel (ELF) in a cohort of patients with chronic hepatitis.

This prospective study was approved by the regional ethics committee. All patients gave fully informed written consent. Forty patients with a clinical indication for liver biopsy were prospectively recruited for liver ECV quantitation at equilibrium CT imaging. Biopsy samples underwent digital image analysis and assessment of collagen content expressed as the collagen-proportionate area (CPA). Spearman correlation was used to evaluate association between ECV, ELF, and CPA. Multiple regression analysis was used to test prediction of CPA by a model that combined ECV and ELF. ECV, ELF score, and CPA were compared with Ishak stage by using the KruskalWallis test.

Complete ECV, ELF, and CPA were available in 33 patients. Liver ECV, CPA, and ELF had a median of 0.26 (interquartile range [IQR], 0.24-0.29), 5.0 (IQR, 3.0-15.0), and 9.71 (IQR, 8.14-10.92), respectively. Hepatic ECV demonstrated good association with CPA $(r=0.64 ; P<$ $.001)$ and ELF score $(r=0.38 ; P<.027)$, with no significant difference in strength of correlation $(P=.177)$. The regression model that combined ELF and ECV achieved good prediction of CPA $\left(R^{2}=0.67 ; P<.001\right)$. Significant variation in ECV and ELF was seen between fibrosis stage groups.

Conclusion: Hepatic ECV measured with equilibrium CT imaging is associated with biopsy-derived CPA and serum ELF-validated markers of liver fibrosis. This suggests that equilibrium CT imaging can quantify diffuse fibrosis in chronic liver disease.

${ }^{\circ}$ RSNA, 2014 
C hronic liver disease is a public health issue (1), and there were an estimated 1.29 million deaths worldwide in 2010 from viral hepatitis alone (2). Regardless of its etiologic cause, sustained liver injury eventually leads to diffuse interstitial fibrosis and expansion of the extracellular space by collagenous scar that distorts the normal architecture, impairs hepatic function, and is the hallmark of cirrhosis. Evaluation of hepatic fibrosis plays a key role in disease management by facilitating prognostication and guiding therapies, such as antiviral agents, which in some instances may reverse the fibrogenic process (3). Histopathologic evaluation of biopsy samples remains the reference standard test for assessment of diffuse hepatic fibrosis, but it has a number of well-documented limitations, including its invasive nature and associated small but significant risk of complications (4). Furthermore, it examines only a tiny fraction of the organ (5) and is therefore prone to sampling error (6). These deficiencies have fueled the development of noninvasive techniques for fibrosis quantification. Liver stiffness measurement with transient elastography is well validated (7), but requires dedicated equipment and is of limited use in obese patients and those with ascites or narrow intercostal spaces (8). Serum biomarkers, such as the enhanced liver fibrosis (ELF) test, are sufficiently related to liver fibrosis to be clinically useful (9), but they are not liver specific (10), and some have other influences, such as renal function, that reduce their discrimination between mild and moderate fibrosis $(11,12)$.

Equilibrium imaging is a technique that uses contrast agents already used

\section{Advance in Knowledge}

- Hepatic extracellular volume measured by using equilibrium contrast agent-enhanced CT imaging shows good association $(r=0.64 ; P<.001)$ with fibrosis morphometrically quantified from histologic sampling in a cohort of patients with chronic viral hepatitis. widely in computed tomographic (CT) and magnetic resonance (MR) imaging to evaluate the fractional extracellular volume (ECV) that is expanded in fibrosis and other deposition processes, such as amyloidosis. The technique with both MR imaging and CT has been applied successfully to quantify myocardial ECV (13-15) as a surrogate marker for diffuse fibrosis. The technique was also applied to the liver by using MR imaging to quantify amyloid deposition (16). Intuitively, the technique may have a role in assessment of diffuse liver fibrosis, and Varenika et al (17) recently described a strong correlation between hepatic ECV and histologic fibrosis area in a rodent model of chronic liver disease. Early retrospective studies (18) suggest that this observation may translate to humans. To our knowledge, there was no validation of equilibrium imaging assessment of diffuse liver fibrosis compared with a histopathologic reference and, in particular, a robust morphometric quantitation of collagen in an appropriate spectrum of patients with a range of disease severity.

The purpose of this initial exploratory study is to prospectively evaluate hepatic ECV measurement by equilibrium CT imaging compared with both fibrosis that was quantified with histologic analysis and the ELF panel (9) in a cohort of patients with chronic hepatitis.

\section{Materials and Methods}

This prospective study was approved by the regional ethics committee (reference number 09/H0716/75). All patients gave fully informed written consent.

Patients were recruited between April 2011 and July 2013 from the chronic liver disease outpatient service at a single tertiary referral center (Royal Free University Hospital, Hampstead, England). Inclusion criteria were serologically confirmed hepatitis B or C, clinically indicated liver

\section{Implication for Patient Care}

- Equilibrium contrast-enhanced CT imaging may provide a useful tool for noninvasive assessment of diffuse hepatic fibrosis. biopsy, and provision of written informed consent for study participation. Exclusion criteria were contraindication to contrast agent-enhanced CT (iodinated contrast agent allergy and estimated glomerular filtration rate $<60 \mathrm{~mL} / \mathrm{min}$ ) or human immunodeficiency virus coinfection, previous liver transplantation, or a history of significant alcohol consumption (>32 $\mathrm{g}$ of alcohol per day for men; $>24 \mathrm{~g}$ of alcohol per for women). There were 190 patients identified who met inclusion criteria. Of these, 32 patients were human immunodeficiency virus positive, 22 patients had a history of liver transplantation, 28 patients had impaired renal function, three patients reported an iodine allergy, and 65 patients had a history of significant alcohol consumption. In total, 40 patients fully met the inclusion and exclusion criteria.

\section{Histologic Analysis}

Liver biopsies were performed according to local guidelines as part of the routine clinical care of the patient. Percutaneous biopsy was performed by using a Menghini technique (19) with a 16-gauge needle from the right lobe. Transjugular biopsy samples were collected with up to four passes of a 19-gauge needle through either the right or middle hepatic vein wall (20).

\section{Published online before print}

10.1148/radiol.14141435 Content codes: GI CT

Radiology 2015; 275:136-143

\section{Abbreviations:}

$\mathrm{CPA}=$ collagen proportionate area

$\mathrm{ECV}=$ extracellular volume fraction

ELF $=$ enhanced liver fibrosis

$\mathrm{IQR}=$ interquartile range

\section{Author contributions:}

Guarantors of integrity of entire study, S.B., J.C.M., S.A.T.; study concepts/study design or data acquisition or data analysis/interpretation, all authors; manuscript drafting or manuscript revision for important intellectual content, all authors; approval of final version of submitted manuscript all authors; agrees to ensure any questions related to the work are appropriately resolved, all authors; literature research, S.B., W.M.R., J.C.M., S.A.T.; clinical studies, S.B., W.M.R., R.J., A.R.H., J.C.M.; experimental studies, S.B., J.C.M.; statistical analysis, S.B., S.P., J.C.M.; and manuscript editing, S.B., S.P., W.M.R., A.D., J.C.M., S.A.T.

Conflicts of interest are listed at the end of this article. 
To ensure reliable histopathologic evaluation, we only included biopsy samples that were $12 \mathrm{~mm}$ or greater in length (21). Samples were formalin fixed, paraffin embedded, and stained with hematoxylin and eosin and Gordon and Sweet staining for reticulin. Fibrosis stage was recorded by an experienced hepatopathologist according to the Ishak system (22). A further tissue section was stained with picroSirius red for collagen-proportionate area (CPA) quantification by digital image analysis. Digital image analysis was performed independent of fibrosis staging by a single operator (A.H.) with the method described by Calvaruso et al (23). A digital camera (Powershot A640; Canon U.S.A., Melville, $\mathrm{NY}$ ) on the macro setting was used for low-magnification whole-section image capture. Digital image analysis was performed by using software (Zeiss KS300; Carl Zeiss Microscopy, Jena, Germany) and included steps to eliminate image artifacts and structural collagen in portal tracts and blood vessel walls. White space within natural structures, such as vascular cavities, was not included.

\section{Equilibrium Contrast-enhanced CT Imaging}

The principal of equilibrium imaging is that contrast agents are exclusively extracellular tracers, and, once they are administered, they passively pass from the blood plasma (intravascular compartment) into the interstitium (extravascular extracellular compartment), but do no not cross cell membranes to enter the cells. During the equilibrium phase after administration of the contrast agent, the concentration of contrast agent within the intravascular compartment is approximately equal to that within the extravascular extracellular compartment. The fractional volume of the extracellular space within a tissue can then be quantified by measuring the relative enhancement of the tissue compared with blood and multiplying by the ECV of blood (1 hematocrit) (15).

In this initial exploratory study, a steady-state contrast agent equilibrium protocol (24) was used based on a bolus injection of contrast agent, followed by an infusion. This method was chosen to minimize potential error that could result from intersubject hemodynamic or contrast agent kinetics variation that could arise from a single bolus technique (13).

Equilibrium CT imaging was performed with a 64-detector row CT scanner (Somatom Sensation 64; Siemens Medical Solutions, Erlangen, Germany) no more than 2 weeks before liver biopsy. Before CT examination, two 5-mL blood samples were collected. The first was sent for complete blood count to obtain blood hematocrit, and the second for ELF analysis.

A scan before administration of the contrast agent was acquired through the upper abdomen to include the whole liver with the following parameters: effective tube current-time product, 250 mAs; pitch, 0.8; tube voltage, $120 \mathrm{kV}$; section collimation, $24 \mathrm{~mm} \times 1.2 \mathrm{~mm}$; gantry rotation time, $500 \mathrm{msec}$; reconstructed to a section thickness of $10 \mathrm{~mm}$ with a soft-tissue convolution kernel (B20f). The iodinated contrast agent iohexol (Omnipaque 300; GE Healthcare, Cork, Ireland) was then administered as a bolus dose of $1 \mathrm{~mL} / \mathrm{kg}$ at $3 \mathrm{~mL} / \mathrm{sec}$; followed immediately by an infusion of $1.88 \mathrm{~mL} / \mathrm{kg}$ per hour based on previous development work (24) that demonstrated contrast agent steady state on sequential plasma iohexol measurements and CT scan attenuation measurements. Mean total contrast agent volume administered across the cohort was $152 \mathrm{~mL} \pm 28$ (standard deviation). After 30 minutes of infusion, the scan volume through the upper abdomen was repeated with the same parameters. Size-specific dose estimates were derived by using scanner-calculated volume CT dose index, and patient anteroposterior and lateral dimensions were measured from the CT series obtained before administration of contrast agent at the midliver level (25).

Image analysis was performed with software (OsiriX v4.1.2; OsiriX Foundation, Geneva, Switzerland) by a radiologist (S.B., with 8 years of experience interpreting body CT scans) who was blinded to biopsy findings.
On three contiguous sections reconstructed to a thickness of $10 \mathrm{~mm}$, a peripherally based wedge-shaped region of interest was drawn in segment VII of the liver (mean area, $34.5 \mathrm{~cm}^{2}$ ) to include the greatest area of liver parenchyma while avoiding visible vessels or incidental hepatic lesions, such as cysts. Because the exact liver segment from which the biopsy samples were collected was not known in all cases, imaging biopsy matching was at the level of the right lobe only. A further elliptical region of interest was drawn on the same section within the abdominal aorta (mean area, $1.6 \mathrm{~cm}^{2}$ ) by avoiding the aortic wall and any atheromatous plaque. Because of the possible uptake of iodinated contrast agent into hepatocytes and vicarious excretion into the biliary system, regions of interest (mean area, $2.8 \mathrm{~cm}^{2}$ ) were also placed in the gallbladder lumen, when present, with visible calcified gallstones avoided. All regions of interest were drawn on the images that were obtained after contrast agent administration and then copied to the matching sections on the images that were obtained before contrast agent administration (Fig 1). Mean attenuation (in Hounsfield units) for liver parenchyma, gall bladder, and blood was derived and liver ECV calculated by using the following formula:

$$
\begin{aligned}
\mathrm{ECV}_{\text {liver }}= & (1-\text { hematocrit }) \\
& \times\left(\Delta \mathrm{HU}_{\text {liver }} / \Delta \mathrm{HU}_{\text {blood }}\right),
\end{aligned}
$$

where $\Delta H U$ are Hounsfield units in equilibrium phase minus Hounsfield units before contrast agent administration.

\section{ELF Test Biomarker}

Patient serum was separated from blood collected before the CT examination and stored at $-80^{\circ} \mathrm{C}$ before it was sent to an independent laboratory (iQur Limited, London, England). Proprietary assays (Siemens Healthcare Diagnostics, Tarrytown, NY) were used in conjunction with an automated analyzer (Advia Centaur; Siemens Healthcare Diagnostics) in accordance with the instructions from the manufacturer to determine levels of tissue inhibitor of matrix metalloproteinase 1 , hyaluronic 
Figure 1
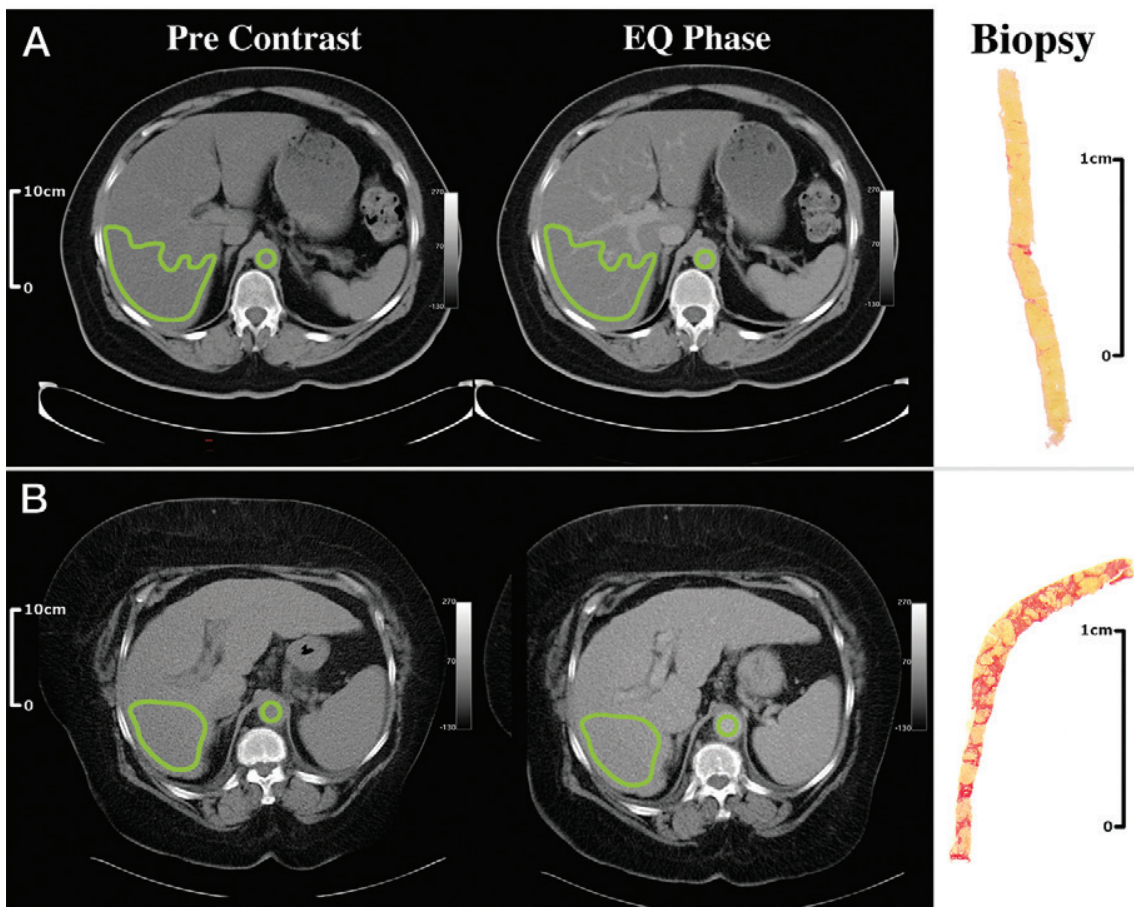

Figure 1: Equilibrium CT and biopsy specimen (picro-Sirius red stain; macro view) images from a patients with, $A$, no significant fibrosis ( $E C V=0.21 ; C P A=3)$ and, $B$, severe fibrosis $(E C V=0.31 ; C P A=24)$.

Sampled volumes between equilibrium (EQ) CT and biopsy differ by magnification of approximately $\times 1500$. Note that the inferior vena cava and right portal vein branches in, $B$, are less visible than in, $A$, because ECV of liver is similar to that of the blood.

acid, and amino-terminal propeptide of type-III collagen. Results were combined by using an algorithm to generate a fibrosis score (9).

\section{Statistical Analysis}

Statistical analyses were performed by using software (Prism 5.0; Graphpad, San Diego, Calif). Kolmogorov-Smirnov analysis was used to test for normality. Non-Gaussian distributed data were expressed as a median and interquartile range. Correlation between variables was evaluated by using Spearman correlation, with one-sided significance testing (set to $P \leq .05$ ), and Fisher $r$-to- $z$ transformation to assess the significance of the difference between correlation coefficients. Linear regression was used to evaluate the ability of ECV, ELF, and a model that combined ECV and ELF scores to predict CPA. The $R$ and $R^{2}$ values were used to assess the strength of correlation, and $P$ values less than .05 were considered to indicate a significant difference.

Histologic stage was divided into low (S0-S1), moderate (S2-S4), and severe (S5-S6) groups, and ECV, CPA, and ELF scores were compared with fibrosis stage by using the Kruskal-Wallis nonparametric analysis of variance test.

A paired $t$ test was used to assess for an increase in attenuation within the lumen of the gallbladder between the before-equilibrium and equilibriumphase images.

\section{Results}

Among the 40 patients who fulfilled the eligibility criteria, complete sets of data (ECV, CPA, and ELF score) were collected in 33 cases (four histologic samples were fragmented or measured less than $12 \mathrm{~mm}$; two patients had estimated

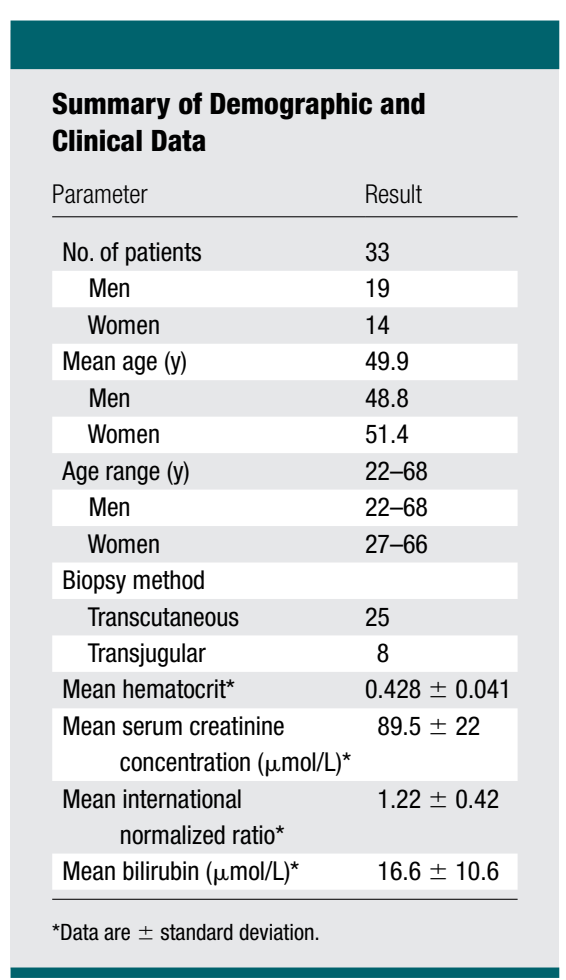

glomerular filtration rates of $<60 \mathrm{~mL} /$ min before undergoing CT examination; and one patient withdrew consent). Among the 33 patients, a diagnosis of hepatitis $\mathrm{C}$ virus infection was obtained in 23 cases and a diagnosis of hepatitis B was obtained in 10 . We collected 24 tissue samples by ultrasonographically guided biopsy and nine tissue samples by transjugular biopsy. Demographic and ELF details are summarized in the Table.

Equilibrium CT examination sizespecific dose estimate had a mean value of $38.8 \mathrm{mGy} \pm 8.2$. The regionof-interest volume was approximately 500 times as large as that of the average of each biopsy $(10.4 \mathrm{~mL}$ vs 0.02 $\mathrm{mL}$ ). The mean attenuation within the liver and aorta before contrast agent administration was $50 \mathrm{HU}$ (range, 24$62 \mathrm{HU}$ ) and $41 \mathrm{HU}$ (range, 32-51 HU), respectively; and at equilibrium phase was $67 \mathrm{HU}$ (range, 38-78 HU) and 80 HU (range, 71-94 HU), respectively.

The gallbladder was present in 24 of the 33 patients analyzed, and mean attenuation within the gallbladder lumen was $9 \mathrm{HU}$ (range, 2-25 HU) before contrast agent administration and 
$11 \mathrm{HU}$ (range, 2-24 HU) after contrast agent administration. Paired $t$ test confirmed no significant difference $(P=$ .08) in gallbladder lumen attenuation between the before contrast agent administration and equilibrium-phase images (mean difference, 1.1 HU).

Median fractional ECV measured in the liver was 0.26 (interquartile range [IQR], 0.24-0.29; range, 0.17-0.47); median CPA was 5.0 (IQR, 3.0-15.0; range, 1.8-28.3); and median ELF score was 9.71 (IQR, 8.14-10.92; range, 7.27-12.43).

Fractional ECV showed good association with $\mathrm{CPA}(r=0.64 ; P<.001)$ and ELF score $(r=0.38 ; P<.027)$ (Fig 2, $A, B$ ), with no significant difference in the strength of the correlations $(P=.177)$. A significant association was also found between ELF score and CPA $(r=0.46 ; P<.007)$ (Fig $2, C)$.

Multiple regression analysis showed that both $\operatorname{ECV}(P<.001)$ and $\operatorname{ELF}(P<$ .006) were significant independent predictors of CPA, with a $t$ statistic for ECV and ELF of 5.20 and 2.94, respectively. The regression model $(80 \times \mathrm{ECV}+1.8$ $\times$ ELF - 30.4) was a better predictor of CPA $\left(R^{2}=0.67 ; P<.001\right)$ (Fig 2,D) than either $\operatorname{ECV}\left(R^{2}=0.56 ; P<.001\right)$ or $\operatorname{ELF}\left(R^{2}=0.35 ; P<.017\right)$ alone.

ECV, ELF, and CPA demonstrated comparable discrimination between Ishak score of S0-S1 versus S5-S6 and S2-S4 versus S5-S6 $(P<.013$, $P<.016$, and $P<.001$, respectively) (Fig $3, A-C$ ). None of the three was able to significantly distinguish S0-S1 and S2-S4 $(P>.05)$.

\section{Discussion}

In this prospective initial exploratory study, we measured ECV by using equilibrium contrast agent CT examination. We found that ECV tracked liver fibrosis, measured either by CPA or by Ishak fibrosis stage. These results are consistent with preliminary studies (17) that use animal models, and they tentatively suggest that a CT examination performed with widely available equipment and contrast agents could be used to evaluate hepatic fibrosis, which would reduce the need for invasive biopsy.
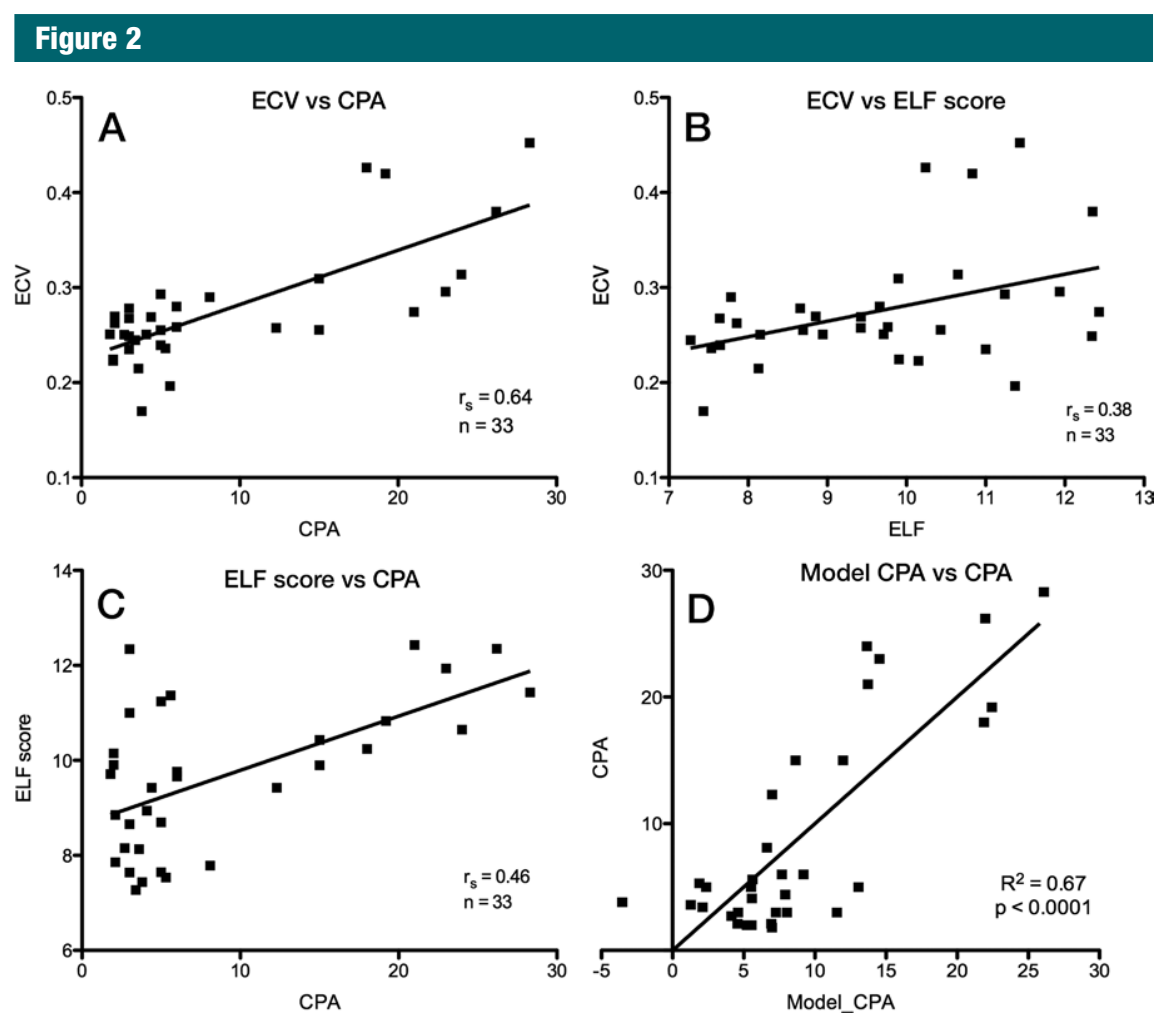

Figure 2: Scatterplots show correlations between ECV measured by using equilibrium $\mathrm{CT}, A$, histologic CPA and, $B$, ELF score, and, $C$, correlation between ELF and CPA, with lines of best fit. $D$, Multiple regression model combines EVC and ELF score to predict CPA.

We used automated morphometric fibrosis quantification as our primary histologic comparator. As a continuous measurement, this largely eliminated limitations of traditional categorical staging. Data suggest that CPA is a better predictor of future clinical decompensation than Ishak stage (26), which shows strong correlation with hepatic venous pressure gradient (a good prognostic index in relation to survival and complications) (27). However, CPA is limited by biopsy, with accuracy dependent on etiologic origin of cirrhosis and biopsy specimen size (21).

We also compared equilibrium CT examination-derived ECV with a biochemical marker: the ELF test. The ELF score is a well-validated marker of hepatic fibrosis that incorporates levels of extracellular matrix components and enzymes involved in their regulation, and it is a strong predictor of clinical outcome in chronic liver disease (28). Previous work showed association between ELF score and histologic fibrosis stage, and this was confirmed by our current study. ECV correlated with ELF, which suggested that ECV does reflect underlying damage and fibrosis, although the strength of association was lower than with CPA. Neither ECV nor ELF has the limitations of histologic staging and CPA, which are imposed by reliance on liver biopsy. It may be that their ability to sample the whole liver volume reduces sampling error and may improve repeatability. Although we primarily used ELF to test the ability of equilibrium CT imaging to estimate liver fibrosis, regression analysis showed them to be independent predictors of CPA, and when ELF and ECV were combined they better predicted CPA than either in isolation. The combination of two noninvasive tests to accurately predict liver fibrosis is clearly an attractive proposition and validation in a new cohort is now required. 


\section{Figure 3}
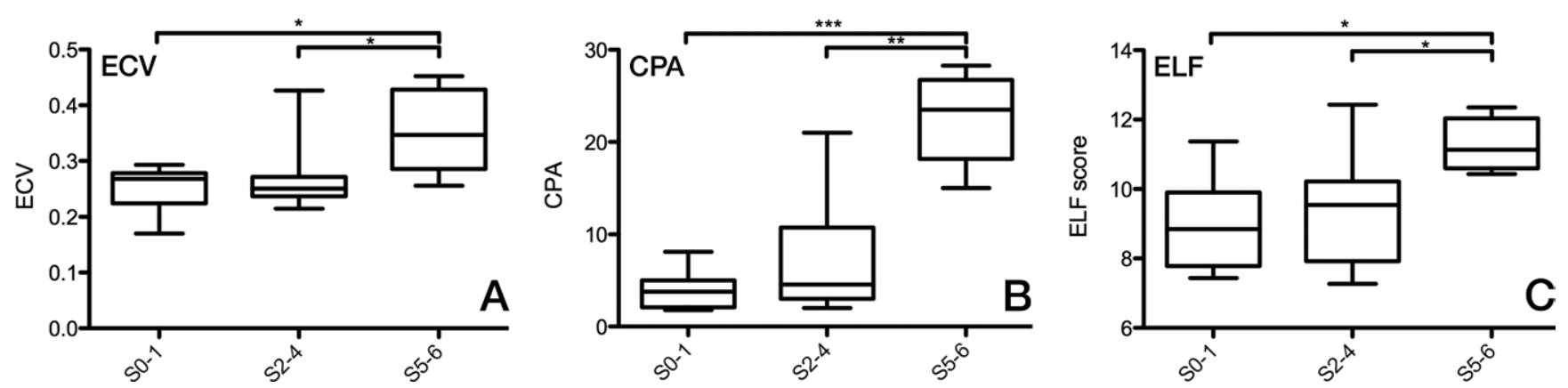

Figure 3: Box-and-whisker plots include median and 25th and 75th percentiles and show, $A, E C V, B, C P A$ and, $C$, ELF score by Ishak fibrosis stage. $* P=.01-.05$, $* * P=.001-.01, * * * P<.001$.

Recent use of equilibrium imaging by several groups provided good evidence for an association between elevated ECV and diffuse fibrosis in the heart $(13,15,29)$, and amyloid protein deposition in the heart, liver, and spleen $(16,14)$. There are, however, limitations of equilibrium imaging for fibrosis assessment. The principle behind its use is that accumulation of extracellular collagenous matrix is accompanied by expansion in the extracellular water into which contrast agents distribute. There are several other processes that may affect this volume and confound fibrosis measurement. Edema from inflammation or venous congestion, for example, may expand ECV, while hepatocellular swelling from steatosis may compress the ECV. A further important point to emphasize is that the ECV includes the ECV in the whole tissue (parenchyma and enclosed vasculature); in other words, both interstitial fluid and the interstitial (plasma) blood compartment. This is small in tissues like myocardium, but much larger and potentially independently varying with disease in the liver. A further potential confounder in the liver is the vicarious uptake of contrast agents by hepatocytes that occurs after delayed contrast agent administration. This phenomenon is thought to be associated with impaired renal function, but has been described in patients with normal kidneys (30). Contrast agent uptake by hepatocytes and excretion via the biliary tree would lead to increased hepatic enhancement relative to blood and an overestimation of liver ECV. The lack of a discernible increase in the attenuation of bile in the gallbladder suggests that in this small group, significant vicarious excretion had not occurred after the 30-minute infusion. It should be noted that patients with major renal impairment were excluded from the study, which therefore lessened the risk of confounding the fractional hepatic ECV estimates by vicarious excretion.

We used standard CT image acquisition and reconstruction techniques, which are widely available. Modern multidetector scanners and reconstruction techniques may reduce image artifacts and noise, leading to more precise and accurate ECV quantitation while reducing the dose of ionizing radiation $(31,32)$. Mean size-specific dose estimate for liver equilibrium CT examination in this study was comparable to that of a standard clinical CT examination of the liver and equivalent to 3.5 years of natural background radiation in the United States (33). The theoretical risk associated with this exposure (34) needs to be weighed against the sampling error and complication risks associated with biopsy and operator dependence and patient-limiting factors associated with transient elastographic imaging.

The reference steady-state equilibrium method used in this study involves establishing a constant plasma concentration of contrast agent via a prolonged infusion. Although time consuming and somewhat impractical, at the time of the study design, it was considered the most robust method. A further limitation of the infusion technique is the relatively low level of enhancement within the aorta and liver during the delayed equilibrium phase. Even with large regions of interest, the contrast-to-noise ratio at this level of enhancement is relatively low. Recently validated equilibrium contrast agent protocols used rapid-bolus-only methods (35) that, for tissues with rapid kinetics (ie, the myocardium), produces a dynamic equilibrium of changing but related plasma and interstitial contrast agent concentrations and may be of sufficient accuracy to replace less practicable protocols that use prolonged infusions. Several groups used this method by imaging before and 7-10 minutes after a standard clinical bolus of contrast material (13), which demonstrated higher contrastto-noise ratio and good approximation of ECV within the myocardium. By using a bolus and infusion technique, we ensured equilibrium; however, our current studies now use this simpler socalled bolus-only technique that allows the whole scan to be performed in 10 minutes. Simple integration of wholeliver ECV quantitation into clinical CT examination, which supplements anatomic assessment and focal lesion detection, is clearly desirable and a future study is now indicated.

There were additional limitations to our study. Our patient selection resulted in a relatively high proportion of patients with mild fibrosis: $70 \%$ of our patient cohort had CPA values clustered between 2 and 8.1 . Future studies 
should endeavor to recruit a patient cohort equally distributed across the range of fibrosis severity. We used a single etiologic origin of virally induced hepatitis to ensure homogeneity in our data. However, we cannot assume the results can be extrapolated to other diseasess, such as alcoholic liver disease and nonalcohoic fatty liver disease.

Factors thought to affect hepatic extracellular volume fraction, such as hepatic inflammation, steatosis, hepatic venous congestion, and portal venous flow, were not controlled for in this study, and future studies may examine the influence of these confounders on hepatic ECV.

Although a comparison is made here with the current reference standard of histologic analysis, we did not include other techniques that showed strong association with clinical markers of liver cirrhosis. Hepatic venous pressure gradient and transient elastography are both well validated by comparison with histologic analysis and are predictive of decompensation and portal hypertension-related complications (36). Future studies should include comparison of ECV with liver stiffness measurement and hepatic venous pressure gradient.

This study provides biopsy-controlled evidence for a relationship between ECV and diffuse liver fibrosis in humans and provides basis for a larger study to examine the diagnostic performance of equilibrium imaging for noninvasive monitoring of liver disease severity. Combination of two noninvasive tests (ECV measured with equilibrium CT imaging and ELF) to accurately predict liver fibrosis is potentially powerful, and validation studies are now indicated.

Acknowledgments: The authors acknowledge the help given to this study by Alison Winstanley, MBBS, BS, MRCPath; Gemma Lewis, BS; and Priti Dutta.

Disclosures of Conflicts of Interest: S.B. disclosed no relevant relationships. S.P. disclosed no relevant relationships. W.M.R. Activities related to the present article: disclosed no relevant relationships. Activities not related to the present article: author reports personal fees from Siemens Healthcare Diagnostics. Other relationships: disclosed no relevant relation- ships. R.J. Activities related to the present article: disclosed no relevant relationships. Activities not related to the present article: reports fees paid to his institution from Ocera Therapeutics for consultancy and patents, Grifols for grants/grants pending, and Norgine for lectures. Other relationships: disclosed no relevant relationships. A.R.H. disclosed no relevant relationships. A.P. disclosed no relevant relationships. J.C.M. disclosed no relevant relationships. S.A.T. Activities related to the pre sent article: disclosed no relevant relationships. Activities not related to the present article: reports personal fees from research consultancy to Robarts. Other relationships: disclosed no relevant relationships.

\section{References}

1. Bosetti C, Levi F, Lucchini F, Zatonski WA, Negri E, La Vecchia C. Worldwide mortality from cirrhosis: an update to 2002 . J Hepatol 2007;46(5):827-839.

2. Cowie BC, Carville KS, MacLachlan JH. Mortality due to viral hepatitis in the Global Burden of Disease Study 2010: new evidence of an urgent global public health priority demanding action. Antivir Ther 2013;18(8):953-954

3. Ellis EL, Mann DA. Clinical evidence for the regression of liver fibrosis. J Hepatol 2012;56(5):1171-1180.

4. Gilmore IT, Burroughs A, Murray-Lyon IM, Williams R, Jenkins D, Hopkins A. Indications, methods, and outcomes of percutaneous liver biopsy in England and Wales: an audit by the British Society of Gastroenterology and the Royal College of Physicians of London. Gut 1995;36(3):437-441.

5. Nord HJ. Biopsy diagnosis of cirrhosis: blind percutaneous versus guided direct vision techniques-a review. Gastrointest Endosc 1982;28(2):102-104.

6. Regev A, Berho M, Jeffers LJ, et al. Sampling error and intraobserver variation in liver biopsy in patients with chronic HCV infection. Am J Gastroenterol 2002;9 $7(10): 2614-2618$.

7. Foucher J, Chanteloup E, Vergniol J, et al. Diagnosis of cirrhosis by transient elastography (FibroScan): a prospective study. Gut 2006;55(3):403-408.

8. Castera L. Noninvasive methods to assess liver disease in patients with hepatitis B or C. Gastroenterology 2012;142(6):12931302.e4.

9. Rosenberg WM, Voelker M, Thiel R, et al. Serum markers detect the presence of liver fibrosis: a cohort study. Gastroenterology 2004;127(6):1704-1713.
10. Abignano G, Cuomo G, Buch MH, et al. The enhanced liver fibrosis test: a clinical grade, validated serum test, biomarker of overall fibrosis in systemic sclerosis. Ann Rheum Dis 2014;73(2):420-427.

11. Wahl K, Rosenberg W, Vaske B, et al Biopsy-controlled liver fibrosis staging using the enhanced liver fibrosis (ELF) score compared to transient elastography. PLoS ONE 2012;7(12):e51906.

12. Parkes J, Guha IN, Roderick P, Rosenberg W. Performance of serum marker panels for liver fibrosis in chronic hepatitis C. J Hepatol 2006;44(3):462-474.

13. Nacif MS, Kawel N, Lee JJ, et al. Interstitial myocardial fibrosis assessed as extra cellular volume fraction with low-radiationdose cardiac CT. Radiology 2012;264(3) 876-883.

14. Banypersad SM, Sado DM, Flett AS, et al. Quantification of myocardial extracellular volume fraction in systemic AL amyloidosis: an equilibrium contrast cardiovascular magnetic resonance study. Circ Cardiovasc Imaging 2013;6(1):34-39.

15. Flett AS, Hayward MP, Ashworth MT, et al. Equilibrium contrast cardiovascular magnetic resonance for the measurement of diffuse myocardial fibrosis: preliminary validation in humans. Circulation 2010;122(2):138-144.

16. Bandula S, Banypersad SM, Sado D, et al. Measurement of tissue interstitial volume in healthy patients and those with amyloidosis with equilibrium contrast-enhanced MR imaging. Radiology 2013;268(3):858-864.

17. Varenika V, Fu Y, Maher JJ, et al. Hepatic fibrosis: evaluation with semiquantitative contrast-enhanced CT. Radiology 2013;266(1):151-158.

18. Zissen MH, Wang ZJ, Yee J, Aslam R, Monto A, Yeh BM. Contrast-enhanced CT quantification of the hepatic fractional extracellular space: correlation with diffuse liver disease severity. AJR Am J Roentgenol 2013;201(6):1204-1210.

19. Menghini G. One-second biopsy of the liver-problems of its clinical application. N Engl J Med 1970;283(11):582-585.

20. Cholongitas E, Burroughs AK. Liver: Tran sjugular liver biopsy yields high-quality samples. Nat Rev Gastroenterol Hepatol 2012; $9(9): 491-492$.

21. Hall AR, Tsochatzis E, Morris R, Burroughs AK, Dhillon AP. Sample size requirement for digital image analysis of collagen proportionate area in cirrhotic livers. Histopathology 2013 62(3):421-430. 
22. Knodell RG, Ishak KG, Black WC, et al. Formulation and application of a numerical scoring system for assessing histological activity in asymptomatic chronic active hepatitis. Hepatology 1981;1(5):431-435.

23. Calvaruso V, Burroughs AK, Standish R, et al. Computer-assisted image analysis of liver collagen: relationship to Ishak scoring and hepatic venous pressure gradient. Hepatology 2009;49(4):1236-1244.

24. Bandula S, White SK, Flett AS, et al. Measurement of myocardial extracellular volume fraction by using equilibrium contrast-enhanced CT: validation against histologic findings. Radiology 2013;269(2):396-403.

25. Christner JA, Braun NN, Jacobsen MC, Carter RE, Kofler JM, McCollough CH. Size-specific dose estimates for adult patients at CT of the torso. Radiology 2012;265 (3):841-847.

26. Calvaruso V, Dhillon AP, Tsochatzis E, et al. Liver collagen proportionate area predicts decompensation in patients with recurrent hepatitis $\mathrm{C}$ virus cirrhosis after liver transplantation. J Gastroenterol Hepatol 2012; 27(7):1227-1232.
27. Armonis A, Patch D, Burroughs A. Hepatic venous pressure measurement: an old test as a new prognostic marker in cirrhosis? Hepatology 1997;25(1):245-248.

28. Parkes J, Roderick P, Harris S, et al. Enhanced liver fibrosis test can predict clinical outcomes in patients with chronic liver disease. Gut 2010;59(9):1245-1251.

29. Wong TC, Piehler K, Meier CG, et al. Association between extracellular matrix expansion quantified by cardiovascular magnetic resonance and short-term mortality. Circulation 2012;126(10):1206-1216.

30. Hopper KD, Weingast G, Rudikoff J, Thickman D. Vicarious excretion of water-soluble contrast media into the gallbladder in patients with normal serum creatinine. Invest Radiol 1988;23(8):604-608.

31. Chen B, Marin D, Richard S, Husarik D, Nelson R, Samei E. Precision of iodine quantification in hepatic CT: effects of iterative reconstruction with various imaging parameters. AJR Am J Roentgenol 2013;200 (5):W475-W482.

32. Shuman WP, Green DE, Busey JM, et al. Model-based iterative reconstruction versus adaptive statistical iterative reconstruction and filtered back projection in liver 64-MDCT: focal lesion detection, lesion conspicuity, and image noise. AJR Am J Roentgenol 2013;200(5):1071-1076.

33. National Council on Radiation Protection and Measurements. Ionizing radiation exposure of the population of the United States. National Council on Radiation Protection report no. 160. Bethesda, Md: National Council on Radiation Protection and Measurements, 2009.

34. Hendee WR, O'Connor MK. Radiation risks of medical imaging: separating fact from fantasy. Radiology 2012;264(2):312-321.

35. White SK, Sado DM, Fontana M, et al. T1 mapping for myocardial extracellular volume measurement by CMR: bolus only versus primed infusion technique. JACC Cardiovasc Imaging 2013;6(9):955-962.

36. Robic MA, Procopet B, Métivier S, et al. Liver stiffness accurately predicts portal hypertension related complications in patients with chronic liver disease: a prospective study. J Hepatol 2011;55(5):1017-1024. 